\title{
Phylogenetic and Variant Analysis of 1,040 SARS-CoV-2 Genomes
}

\author{
Eric C. Rouchka ${ }^{1,2^{*}}$, Julia H. Chariker2,3, Donghoon Chung ${ }^{4}$ \\ 1Department of Computer Science and Engineering, University of Louisville, Louisville, Kentucky USA \\ ${ }^{2}$ Kentucky Biomedical Research Infrastructure Network Bioinformatics Core, University of Louisville, Louisville, \\ Kentucky USA \\ ${ }^{3}$ Neuroscience Training Program, University of Louisville, Louisville, Kentucky USA \\ ${ }^{4}$ Department of Microbiology and Immunology, University of Louisville, Louisville, Kentucky USA \\ ${ }^{*}$ Corresponding Author \\ eric.rouchka@louisville.edu
}

Keywords: SARS-CoV-2, COVID-19, coronavirus, variant analysis, phylogenetic analysis, viral evolution

\begin{abstract}
The severe acute respiratory syndrome-coronavirus 2 (SARS-CoV-2) viral genome is an RNA virus consisting of approximately 30,000 bases. As part of testing efforts, whole genome sequencing of human isolates has resulted in over 1,600 complete genomes publicly available from GenBank. We have performed a comparative phylogenetic analysis of the sequences, in order to detect common mutations within the population. Analysis of variants occurring within the assembled genomes yields 417 variants occurring in at least $1 \%$ of the completed genomes, including 229 within the 5' untranslated region (UTR), 152 within the 3'UTR, 2 within intergenic regions and 34 within coding sequences.
\end{abstract}

\section{Introduction}

SARS-CoV-2, formerly known as the "Wuhan seafood market pneumonia virus," is a novel coronavirus that first appeared at the seafood and wildlife wholesale market in Wuhan, Hubei Provence, China during late November/early December, 2019 (Zhu et al., 2020). Due to its high human-to-human transmission rate $(\mathrm{Li}$ et al., 2020a), longer than normal latent period (Bar-On et al., 2020) and mortality rates in vulnerable populations, a global pandemic was declared by the World Health Organization (WHO) on March 11, 2020 for the associated COVID-19 disease (Cohen and Kupferschmidt, 2020). As of May 10, 2020, a total of 4,006,257 cases resulting in 278,892 deaths in 215 countries have been confirmed (World Health Organization, 2020).

The reference genome of the SARS-Cov-2 RNA virus (GenBank accession NC_045512) consists of 29,903 bases. Among its features are a 265 base 5' untranslated region (UTR) and a 3' UTR composed of 229 bases. Its coding regions consist of 10 open reading frames (ORFs) coding for 26 genes (Bar-On et al., 2020), including 13,218 bases coding for the ORF1ab polyproteins, whose transcription includes a 1 bp ribosomal slippage event (Atkins et al.,
2016); 3,822 bases coding for a spike surface glycoprotein (S), 228 bases coding a small envelope protein (E), 669 bases coding for a membrane glycoprotein protein (M) and 1,260 bases coding for a nucleocapsid protein $(\mathrm{N})$ along with five additional ORFs (ORF3a, ORF6, ORF7a/ORF7b, ORF8, and ORF10) (Figure 1).

Since the public release of the first reference sequence (MN908947; NC_045512) from NCBI's GenBank (Sayers et al., 2019) on January 12, 2020, the number of sequences available has increased exponentially, at a current rate of approximately 70 new sequences per day (Figure 2). Isolates have been sequenced from 27 countries (Table 1), as well as 34 states, Washington DC, and passengers from a cruise (Table 2).

SARS-CoV-2 is postulated to have originated from zoonotic transfer of a pangolin betacoronavirus based on a phylogenetic analysis of coronavirus sequences, due to a common insertion of 12 nucleotides within the receptor binding domain of the $\mathrm{S}$ protein region that optimizes binding to the human ACE2 receptor, although the most similar betacoronavirus is the bat RaTG13 (Andersen et al., 2020). RNA viruses are characterized by a high mutation rate (Duffy, 2018) driven by RNA dependent RNA 


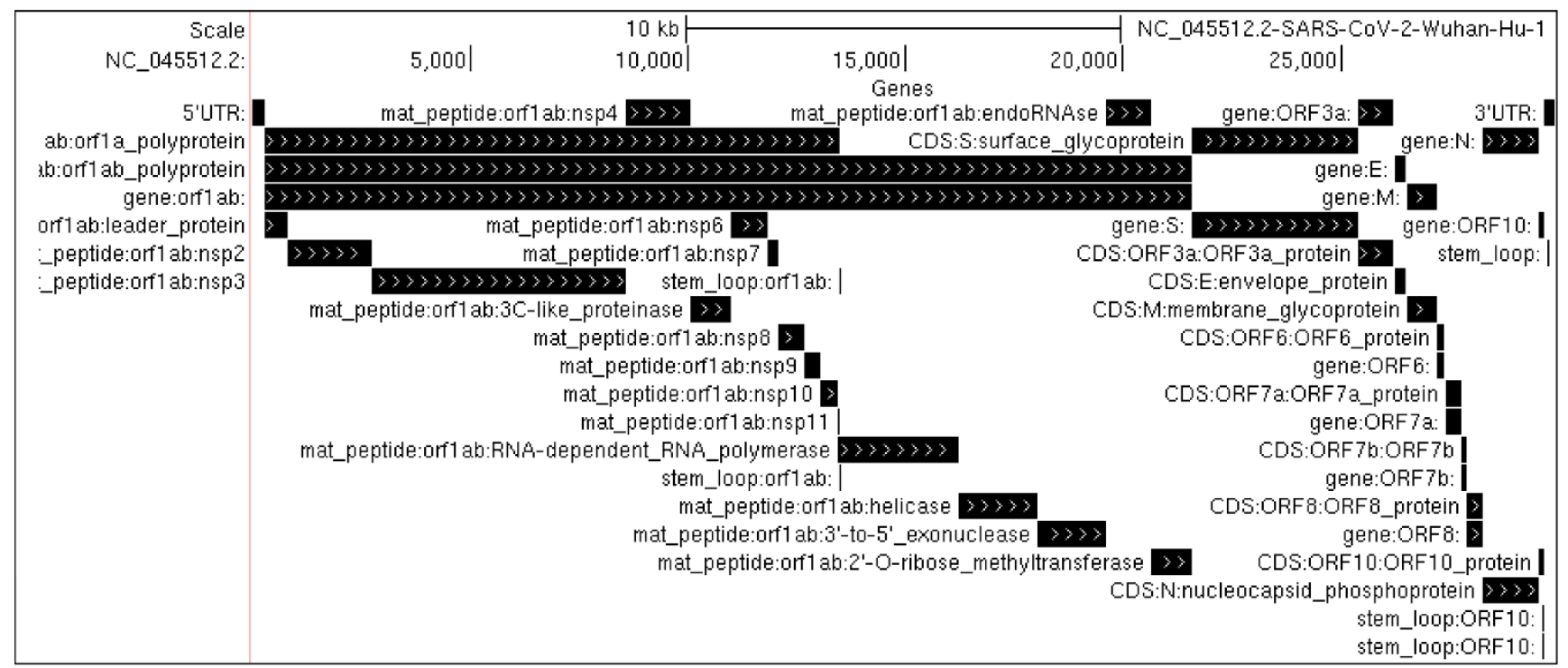

Figure 1: Genome structure of SARS-CoV-2. Shown are the locations of the 10 major open reading frames, as well as specific peptides and structural elements produced within them.

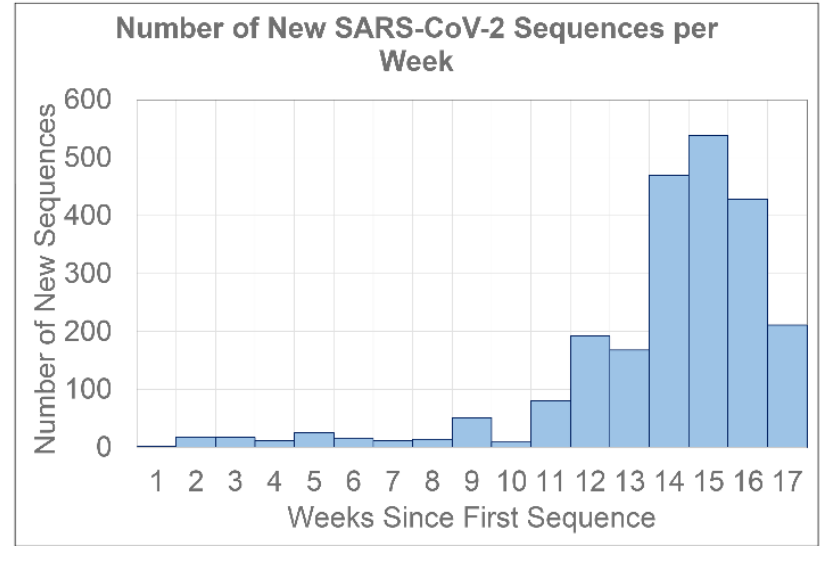

Figure 2: Number of new SARS-CoV-2 sequences deposited in GenBank on a weekly basis through May $7, \mathbf{2 0 2 0}$. Note that week 17 is only a partial week.

Table 1: Number of SARS-CoV-2 sequences in GenBank by country.

\begin{tabular}{lr}
\hline Country & Count \\
\hline USA & 1025 \\
CHN & 64 \\
TWN & 21 \\
HKG & 19 \\
IND & 10 \\
ESP & 7 \\
PRI & 5 \\
GRC & 4 \\
KAZ & 4 \\
KOR & 4 \\
LKA & 4 \\
BRA & 2 \\
ISR & 2 \\
\hline
\end{tabular}

\begin{tabular}{lr}
\hline Country & Count \\
\hline MYS & 2 \\
PAK & 2 \\
VNM & 2 \\
AUS & 1 \\
FRA & 1 \\
IRN & 1 \\
ITA & 1 \\
NPL & 1 \\
PER & 1 \\
SWE & 1 \\
TUR & 1 \\
ZAF & 1 \\
& \\
\hline
\end{tabular}

polymerase $(\mathrm{RdRp})$ that results in viral evolution (Pachetti et al., 2020). Given the importance of the genome sequence with host transmission (in particular the $\mathrm{S}$ protein), it is important to understand common mutations within the population in order to have a better handle on viral load, virus spread, virus evolution, and disease severity.
Table 2: Number of SARS-CoV-2 sequences in GenBank by state/region.

\begin{tabular}{lr}
\hline State/Region & Count \\
\hline USA-WA & 334 \\
USA-NY & 185 \\
USA-CA & 115 \\
USA-MI & 44 \\
USA-VA & 37 \\
USA-CT & 28 \\
USA-CruiseA & 25 \\
USA-ID & 22 \\
USA-MA & 19 \\
USA-GA & 15 \\
USA-FL & 10 \\
USA-UT & 10 \\
USA-WI & 10 \\
USA-PA & 9 \\
USA-IA & 7 \\
USA-IL & 7 \\
USA-MN & 7 \\
USA-NC & 7 \\
\hline
\end{tabular}

\begin{tabular}{lr}
\hline State/Region & Count \\
\hline USA-SC & 7 \\
USA-AZ & 6 \\
USA-TX & 5 \\
USA-IN & 4 \\
USA-LA & 4 \\
USA-NH & 4 \\
USA-OR & 4 \\
USA-RI & 4 \\
USA-KY & 3 \\
USA-NJ & 3 \\
USA-OH & 3 \\
USA-HI & 2 \\
USA-MD & 2 \\
USA-NE & 2 \\
USA-NV & 2 \\
USA-DC & 1 \\
USA-KS & 1 \\
USA-MO & 1 \\
\hline
\end{tabular}

A number of previous studies have examined variants within SARS-CoV-2 isolates. One prior study looking at the genome diversity of SARS-CoV-2 have identified 93 mutations occurring in at least one isolate from a set of 86 complete genomes (Phan, 2020). A second report from a set of 220 complete genomes identified eight novel recurring mutations, with specific prevalence within Asian, North American, and European populations (Pachetti et al., 2020). This study by Pachetti, et al., also showed the occurrence of mutations over time based on sequence sampling dates. A report by Yin (Yin, 2020) identified fifteen highfrequency single nucleotide polymorphisms when comparing a set of 558 SARS-CoV-2 strains. This study found four of these mutations, $241 \mathrm{C}>\mathrm{T}, 3037 \mathrm{C}>\mathrm{T}$, $14408 \mathrm{C}>\mathrm{T}$, and $23403 \mathrm{~A}>\mathrm{G}$ to be more prevalent in European strains, where the COVID-19 is typically more severe. Wang et al. (Wang et al., 2020a) detected 13 variation sites among 95 full-length genomic sequences (variants occurring in at least 3 isolates), with two at 
positions 8,782 and 28,144 showing a high mutation rate around $30 \%$. Khailany et al. (Khailany et al., 2020) looked at mutations within 95 complete genome sequences, and found 116 mutations occurring in at least one isolate, with the most common being $8762 \mathrm{C}>\mathrm{T}, 28144 \mathrm{~T}>\mathrm{C}$, and 29095C > T. Tang et al. (Tang et al., 2020) studied mutations across 103 strains, and determined there were mutations in 149 sites, including six nonsynonymous mutations occurring at least twice. Additionally, this study identified two different mutations (with near complete linkage between $8782 \mathrm{~T}>\mathrm{C}$ and $28144 \mathrm{C}>\mathrm{T}$ ) that separated the virus into two groups labeled L and S. Using deep sequencing reads, Tang et al. also identified 18 locations showing intrahost variants, demonstrating heterogeneity of the virus within a specific host. Wang et al. (Wang et al., 2020b) identified ten high frequency mutations within a set of 108 isolates, which they say can be used to classify SARS-CoV-2 into five main groups. The mutations published include 28144T $>\mathrm{C}, 8782 \mathrm{C}>\mathrm{T}, 23403 \mathrm{~A}>\mathrm{G}$, $3037 \mathrm{C}>\mathrm{T}, 11083 \mathrm{G}>\mathrm{T}, 26144 \mathrm{G}>\mathrm{T}$, and $2261 \mathrm{G}>\mathrm{T}$.

Forster et al. (Forster et al., 2020) used phylogenetic network analysis to find three central variants, A, B, and C, distinguishing East Asian isolates from European and American isolates. In their study, variants found defined clusters and/or subclusters by synonymous mutations 29095T > C (subclustering A), synonymous mutation $8782 \mathrm{~T}>\mathrm{C}$ and nonsynonymous mutation $28144 \mathrm{C}>\mathrm{T}$ (separation of clusters A and B), In addition, Shen et al. (Shen et al., 2020) observed a median of 1-4 intrahost variants, ranging from $0-51$, from a set of eight patients infected with SARS-CoV-2. Included in the Shen study were two patients from the same household, one of which was likely to have infected the other. Interestingly, only $7 / 25$ variants detected in these two individuals were shared, illustrating the high viral mutation rate.

The SARS-CoV-2 spike (S) protein is of particular interest, due to its interaction with the human ACE2 protein that helps to mediate infection of host cells. Korber, et al. (Korber et al., 2020) have set up a workflow for measuring the dynamics of nonsynonymous mutations within the $\mathrm{S}$ protein coding region. This study has uncovered a number of amino acid mutations in the S protein, including D614G $(23403 \mathrm{G}>\mathrm{A})$, which is thought to increase transmissibility due to its rapid expansion in global samples. This mutation was shown to have a high association with two other mutations, 3037C $>\mathrm{T}$ and $14409 \mathrm{C}>\mathrm{T}$.

\section{Materials and Methods}

All available SARS-CoV-2 nucleotide sequences and associated annotations (including locality) were downloaded from NCBI (https://www.ncbi.nlm.nih.gov/genbank/sars-cov-2-seqs/) on 5/7/2020, resulting in 2,262 sequences. Geographical location information was reduced to the corresponding ISO 3166-1 alpha-3 country code (Supplemental Table 1), and the two-letter abbreviated state code. Sequences were then filtered to include only those listed as complete with an isolation source of "Homo sapiens", which left 1,695 sequences listed as complete genomes isolated from human samples. Sequences from this set containing gaps in their assembly (defined by the character "N") were removed from further analysis. Isolates that were $100 \%$ matches across their entire length were merged into a single representative sequence (Table 3). A final total of 1,043 sequences were used in the final analysis, including three from Kentucky that we previously analyzed (Chung et al., 2020). These sequences were then compared against each other, and a distance matrix was constructed based on the number of gaps and mismatches, as calculated by NCBI blastn (v2.10.0) (Johnson et al., 2008). A multiple sequence alignment was performed using kalign (v3.2.5) (Lassmann, 2020) which resulted in a .aln alignment file.

\section{$3 \quad$ Results}

Our analysis focused on a set of 1,043 filtered sequences, including 1,040 publicly available in GenBank, as well as three new isolates sequenced by our group in Kentucky (GenBank accessions MT365025, MT365026, and MT365027). From our group of sequences, we found 47 groups of sequences that had at least two isolates that were $100 \%$ identical, including one group with 18 isolates all from Washington state; a second group with 16 isolates primarily from Washington state, and one group of 14 isolates all from Zhejiang, China (Table 3). These clustered groups of sequences are not surprising, particularly when examining the geographical similarities, suggesting clusters of similar transmission in both time and viral strains. The majority of the sequences were from the United States (Table 1, Figure 3), with California, New York, and Washington comprising the majority of the sequences (Table 2, Figure 4).

Based on a variant threshold of minor allele frequencies of $1 \%$ or more, we detected a total of 417 locations where isolate genomes express an alternate allele other than the reference, NC_045512, including 229 in the 5'UTR, 21 in

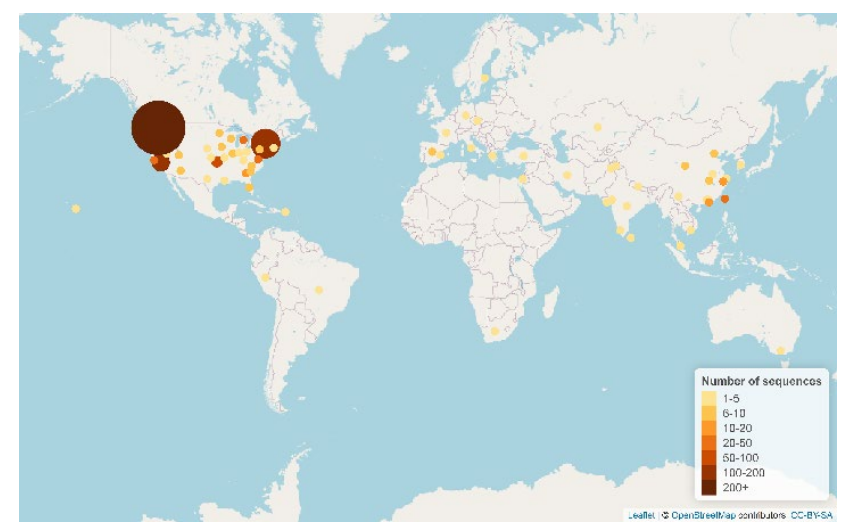

Figure 3: Distribution of the number of SARS-CoV-2 sequences in GenBank worldwide. 
Table 3: Sequence groups with isolates sharing $100 \%$ identity.

\begin{tabular}{|c|c|c|c|}
\hline Name & $\begin{array}{l}\text { Num } \\
\text { Seqs }\end{array}$ & Location & Other Locations \\
\hline MULTIPLE_31 & 18 & USA-WA & -- \\
\hline MULTIPLE_25 & 16 & USA-WA & $\begin{array}{l}\text { USA-NY,USA-NC,USA- } \\
\text { UT }\end{array}$ \\
\hline MULTIPLE_9 & 14 & $\begin{array}{l}\text { CHN- } \\
\text { Zhejiang }\end{array}$ & -- \\
\hline MULTIPLE_8 & 13 & USA-MA & $\begin{array}{l}\text { USA-KS,USA-NC,USA- } \\
\text { PA }\end{array}$ \\
\hline MULTIPLE_21 & 12 & USA-NY & -- \\
\hline MULTIPLE_11 & 9 & $\begin{array}{l}\text { USA- } \\
\text { CruiseA }\end{array}$ & USA-IL \\
\hline MULTIPLE_16 & 7 & USA-GA & USA-SC \\
\hline MULTIPLE_26 & 7 & USA-NY & -- \\
\hline MULTIPLE_35 & 5 & USA-NY & -- \\
\hline MULTIPLE_6 & 4 & USA-MA & -- \\
\hline MULTIPLE_12 & 4 & USA-IA & -- \\
\hline MULTIPLE_45 & 4 & USA-NY & USA-RI,USA-OH \\
\hline MULTIPLE_2 & 3 & USA-MI & -- \\
\hline MULTIPLE_3 & 3 & USA-NY & -- \\
\hline MULTIPLE_-4 & 3 & HKG & -- \\
\hline MULTIPLE_10 & 3 & KOR & -- \\
\hline MULTIPLE_18 & 3 & USA-NY & -- \\
\hline MULTIPLE_-23 & 3 & USA-NY & USA-NJ \\
\hline MULTIPLE_24 & 3 & USA-OH & USA-PA \\
\hline MULTIPLE_28 & 3 & CZE & -- \\
\hline MULTIPLE_29 & 3 & HKG & -- \\
\hline MULTIPLE_33 & 3 & USA-NH & -- \\
\hline MULTIPLE_37 & 3 & USA-NY & -- \\
\hline MULTIPLE_40 & 3 & USA-WA & -- \\
\hline MULTIPLE_43 & 3 & $\begin{array}{l}\text { CHN- } \\
\text { Beijing }\end{array}$ & -- \\
\hline MULTIPLE_1 & 2 & USA & USA-CA,USA-WA \\
\hline MULTIPLE_5 & 2 & USA-NY & -- \\
\hline MULTIPLE_7 & 2 & $\mathrm{CHN}$ & -- \\
\hline MULTIPLE_13 & 2 & USA-CA & -- \\
\hline MULTIPLE_14 & 2 & -- & USA-CA,TWN \\
\hline MULTIPLE_15 & 2 & USA-IN & -- \\
\hline MULTIPLE_17 & 2 & USA-IA & -- \\
\hline MULTIPLE_19 & 2 & USA-WA & -- \\
\hline MULTIPLE_20 & 2 & $\mathrm{CHN}$ & -- \\
\hline MULTIPLE_22 & 2 & TWN & -- \\
\hline MULTIPLE_27 & 2 & USA-NY & -- \\
\hline MULTIPLE_30 & 2 & USA-NY & -- \\
\hline MULTIPLE_32 & 2 & USA-FL & -- \\
\hline MULTIPLE_34 & 2 & ESP & -- \\
\hline MULTIPLE_36 & 2 & USA-NE & -- \\
\hline MULTIPLE_38 & 2 & USA-CA & -- \\
\hline MULTIPLE_39 & 2 & USA-MI & -- \\
\hline MULTIPLE_41 & 2 & USA-WA & -- \\
\hline MULTIPLE_42 & 2 & USA & -- \\
\hline MULTIPLE_44 & 2 & USA-CA & -- \\
\hline MULTIPLE_46 & 2 & -- & USA-PA,USA-VA \\
\hline MULTIPLE_47 & 2 & USA-NY & -- \\
\hline
\end{tabular}

ORF1ab, 2 in S, 3 in ORF3a, 1 in M, 3 in ORF8, 4 in N, 2 intergenic, and 152 in the 3 'UTR. The vast majority of the detected variants lie within the 5'UTR and 3'UTR, and represent deletion events. Since these may be due to a number of factors such as sequencing preparation (i.e. selection of amplicon primers) and difficulty in multiple sequence alignment construction that contribute to less reliability, we only retained seven of the UTR variants for further consideration. This filtering led to a total of 44 variants (Table 4). Of these, twenty have previously been reported (Supplemental Table 2), including the pair at locations $8782 / 28144$ which was previously demonstrated to have a high linkage (Tang et al., 2020) and the tuples

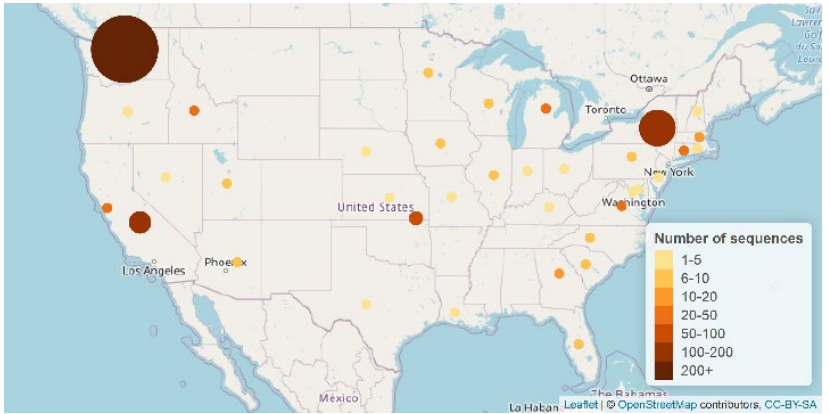

Figure 4: Distribution of the number of SARS-CoV-2 sequences in the United States available in GenBank.

at locations 8782/18060/28144, 241/3037/23403/28144, and $241 / 3037 / 14408 / 23403$ which were shown to have a high number of descendants (Yin, 2020). We uncovered 24 novel variants not previously described to our knowledge, which may be a result of more recent mutation events and/or fixation of a mutation within the population.

Surprisingly, two of these, $1059 \mathrm{C}>\mathrm{T}$ in the nsp2 region of ORF1ab and $25563 \mathrm{G}>\mathrm{T}$ in ORF3a are found at a high frequency within the population, at a rate of $43 \%$ and $49 \%$, respectively.

Linkage between the detected variants was analyzed using Haploview (v4.2) (Barrett et al., 2005) (Figures 5 and 6).

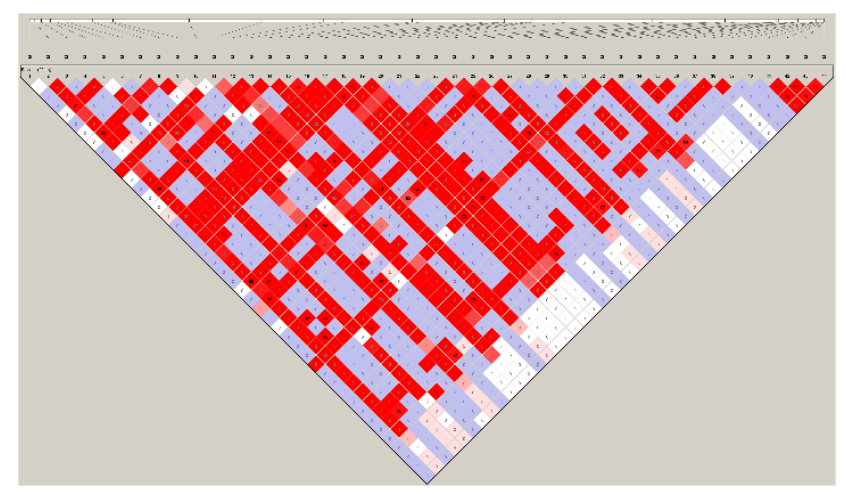

Figure 5: Linkage disequilibrium between SARS-CoV-2 variants. Shown is the Haploview pairwise association between variants. The colors are determined based on the default D'/LOD score. The value shown in each cell is the $r^{2}$ value.

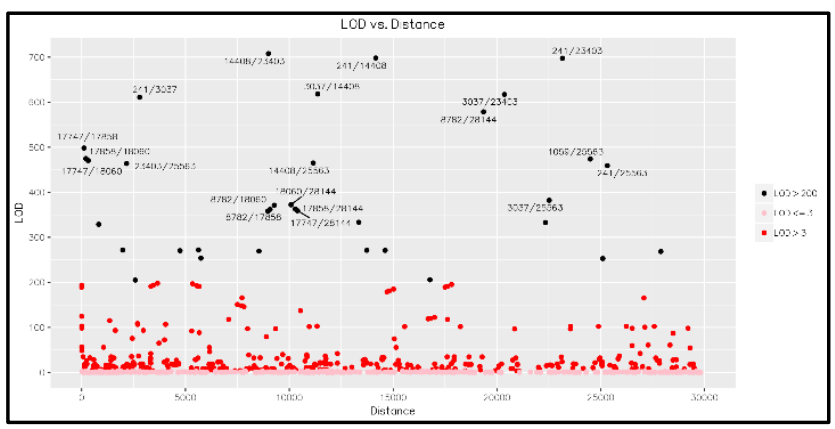

Figure 6: LOD of each variant pair vs. their distance. Points in black represent pairs with a LOD score $>200$. The twenty pairs with the highest LOD scores are labeled based on their positions within the reference genome. 
Table 4: Common variants $(>1 \%)$ detected within SARS-CoV-2 isolates.

\begin{tabular}{|c|c|c|c|c|c|c|c|c|c|c|c|}
\hline \multirow{2}{*}{$\begin{array}{c}\text { Ref } \\
\text { Position }\end{array}$} & \multirow[b]{2}{*}{ Region } & \multirow{2}{*}{$\begin{array}{c}\text { Alignment } \\
\text { Frequencies }\end{array}$} & \multicolumn{3}{|c|}{ Nucleotide } & \multicolumn{3}{|c|}{ Codon } & \multicolumn{3}{|c|}{ Amino Acid } \\
\hline & & & Cons & Alt & Ref & Cons & Alt & Ref & Cons & Alt & Ref \\
\hline 50 & 5'UTR & C:811;-:220;T:10;A:2 & $\mathrm{C}$ & $\bar{T}$ & C & NA & NA & NA & NA & NA & NA \\
\hline 241 & 5'UTR & T:599;C:436;-:7;Y:1 & $\mathrm{T}$ & C & C & NA & NA & NA & NA & NA & NA \\
\hline 490 & ORF1ab1 & $\mathrm{T}: 1022 ; \mathrm{A}: 20 ; \mathrm{W}: 1$ & $\mathrm{~T}$ & A & $\mathrm{T}$ & GAT & GAA & GAT & Asp & Glu & Asp \\
\hline 833 & ORF1ab1 & $\mathrm{T}: 1011 ; \mathrm{C}: 32$ & T & C & $\mathrm{T}$ & TTC & CTC & TTC & Phe & Leu & Phe \\
\hline 1059 & ORF1ab1 & C:593;T:449;Y:1 & C & $\mathrm{T}$ & C & ACC & ATC & ACC & Thr & Ile & Thr \\
\hline 1397 & ORF1ab1 & $G: 1030 ; A: 13$ & G & $A$ & G & GTA & ATA & GTA & Val & Ile & Val \\
\hline 2416 & ORF1ab1 & C:1023;T:20 & C & $\mathrm{T}$ & C & TAC & TAT & TAC & Tyr & Tyr & Tyr \\
\hline 3037 & ORF1ab1 & T:586;C:457 & $\mathrm{T}$ & C & C & TTT & TTC & TTC & Phe & Phe & Phe \\
\hline 3177 & ORF1ab1 & C:1022;T:20;Y:1 & C & $\mathrm{T}$ & C & CCT & CTT & CCT & Pro & Leu & Pro \\
\hline 6040 & ORF1ab1 & C:1028;T:15 & C & $\mathrm{T}$ & C & TTC & TTT & TTC & Phe & Phe & Phe \\
\hline 8782 & ORF1ab1 & C:791;T:251;Y:1 & C & $\mathrm{T}$ & C & AGC & AGT & $A G C$ & Ser & Ser & Ser \\
\hline 11083 & ORF1ab1 & G:973;T:69;C:1 & G & $\mathrm{T}$ & G & TTG & TTT & TTG & Leu & Phe & Leu \\
\hline 11916 & ORF1ab1 & C:1008;T:35 & C & $\mathrm{T}$ & C & TCA & TTA & TCA & Ser & Leu & Ser \\
\hline 14408 & ORF1ab2 & T:603;C:440 & $\mathrm{T}$ & C & C & CTT & CCT & CCT & Leu & Pro & Pro \\
\hline 14805 & ORF1ab2 & C:1003;T:40 & C & $\mathrm{T}$ & C & TAC & TAT & TAC & Tyr & Tyr & Tyr \\
\hline 17247 & ORF1ab2 & $\mathrm{T}: 1018 ; \mathrm{C}: 25$ & T & C & T & CGT & CGC & CGT & Arg & Arg & Arg \\
\hline 17747 & ORF1ab2 & C:850;T:189;Y:4 & C & $\mathrm{T}$ & C & ССТ & CTT & ССТ & Pro & Leu & Pro \\
\hline 17858 & ORF1ab2 & A:851;G:192 & A & G & A & TAT & TGT & TAT & Tyr & Cys & Tyr \\
\hline 18060 & ORF1ab2 & C:849;T:194 & C & $\mathrm{T}$ & C & CTC & CTT & CTC & Leu & Leu & Leu \\
\hline 18736 & ORF1ab2 & T:1024;C:19 & T & C & T & TTT & CTT & TTT & Phe & Leu & Phe \\
\hline 18877 & ORF1ab2 & C:1004;T:39 & C & $\mathrm{T}$ & C & CTA & TTA & CTA & Leu & Leu & Leu \\
\hline 18998 & ORF1ab2 & C:1017;T:26 & C & $\mathrm{T}$ & C & GCA & GTA & GCA & Ala & Val & Ala \\
\hline 20268 & ORF1ab2 & $\mathrm{A}: 1031 ; \mathrm{G}: 12$ & A & G & A & TTA & TTG & TTA & Leu & Leu & Leu \\
\hline 23403 & S & G:602;A:439;R:2 & G & A & A & GGT & GAT & GAT & Gly & Asp & Asp \\
\hline 24034 & $\mathrm{~s}$ & C:1017;T:25;Y:1 & C & $\mathrm{T}$ & C & AAC & AAT & AAC & Asn & Asn & Asn \\
\hline 25563 & ORF3a & G:536;T:507 & G & $\mathrm{T}$ & G & CAG & CAT & CAG & Gln & His & Gln \\
\hline 25692 & ORF3a & C:1022;T:21 & C & $\mathrm{T}$ & C & GGC & GGT & GGC & Gly & Gly & Gly \\
\hline 26144 & ORF3a & G:999;T:44 & G & $\mathrm{T}$ & G & GGT & GTT & GGT & Gly & Val & Gly \\
\hline 26729 & M & $\mathrm{T}: 1020 ; \mathrm{C}: 22 ; \mathrm{Y}: 1$ & T & C & $\mathrm{T}$ & GCT & GCC & GCT & Ala & Ala & Ala \\
\hline 27964 & ORF8 & C:990;T:52;-:1 & C & $\mathrm{T}$ & C & TCA & TTA & TCA & Ser & Leu & Ser \\
\hline 28077 & ORF8 & G:1017;C:22;T:2;-:1;S:1 & G & C & G & GTG & CTG & GTG & Val & Leu & Val \\
\hline 28144 & ORF8 & T:791;C:250;-:1;Y:1 & $\mathrm{T}$ & C & $\mathrm{T}$ & TTA & TCA & TTA & Leu & Ser & Leu \\
\hline 28688 & $\mathrm{~N}$ & $\mathrm{~T}: 1032 ; \mathrm{C}: 11$ & $\mathrm{~T}$ & C & $\mathrm{T}$ & TTG & CTG & TTG & Leu & Leu & Leu \\
\hline 28881 & $\mathrm{~N}$ & G:990;A:53 & G & A & G & AGG & AAG & AGG & Arg & Lys & Arg \\
\hline 28882 & $\mathrm{~N}$ & G:991;A:52 & G & A & G & AGG & AGA & AGG & Arg & Arg & Arg \\
\hline 28883 & $\mathrm{~N}$ & G:991;C:52 & G & C & G & GGA & CGA & GGA & Gly & Arg & Gly \\
\hline 29540 & INTERGENIC & G:1017;A:26 & G & A & G & NA & NA & NA & NA & NA & NA \\
\hline 29553 & INTERGENIC & G:939;A:104 & G & A & G & NA & NA & NA & NA & NA & NA \\
\hline 29700 & 3'UTR & $A: 1023 ; G: 20$ & A & G & A & NA & NA & NA & NA & NA & NA \\
\hline 29742 & 3'UTR & G:1025;T:11;A:4;-:3 & G & $\mathrm{T}$ & G & NA & NA & NA & NA & NA & NA \\
\hline 29864 & 3'UTR & G:746;-:275;A:19;T:1;R:1;V:1 & G & A & G & NA & NA & NA & NA & NA & NA \\
\hline 29867 & 3'UTR & T:645;-:361;A:37 & $\mathrm{T}$ & A & $\mathrm{T}$ & NA & NA & NA & NA & NA & NA \\
\hline 29868 & 3'UTR & G:625;-:367;A:39;C:8;R:3;D:1 & G & A & G & NA & NA & NA & NA & NA & NA \\
\hline 29870 & 3'UTR & C:608;-:378;A:50;M:5;H:1;T:1 & C & A & C & NA & NA & NA & NA & NA & NA \\
\hline
\end{tabular}

Cons: alignment consensus; Alt: alignment alternative; Ref: NC_ 045512 reference sequence. Note that the ORF1ab open reading frame is split into two parts, ORF1ab1 which occurs from bases $266 \ldots 13468$ in the reference sequence, and ORF1ab2 which occurs from bases $13468 \ldots 21555$ as result of a polymerase slippage event.

A total of 38 associations with an $\mathrm{r}^{2}$ value $>=0.8$ were detected (Table 5). In order to further examine these associations for viral evolution, we looked at their frequencies within geographic groupings, including China, East Asia, Hong Kong and Taiwan (Figure 7); China, India, and West Asia (Figure 8); and China, Cruise A, USA, and Europe (Figure 9), as well as by sampling date by month, ranging from December, 2019 to April, 2020 (Figure 10). Intriguingly, the time analysis shows nine variants that are shifting frequency in samples more recently procured during March-April 2020, including $490 \mathrm{~T}>\mathrm{A}, 1397 \mathrm{G}>\mathrm{A}, 23403 \mathrm{~A}>\mathrm{G}$, and $29540 \mathrm{G}>\mathrm{A}$. Eight of these variants are in coding regions, with six producing non-synonymous mutations in the amino acid sequence. One of these, 23403A $>\mathrm{G}$ has been recently reported as a mutation in the Spike protein resulting in a more transmissible form of SARS-CoV-2 (Korber et al., 2020).

\section{Discussion}

Of the 44 common variants we found, 20 result in nonsynonymous mutations which may have functional relevance. Understanding these specific variants is critical to being able to react to the evolution of SARS-CoV-2, in particular, in developing an effective vaccine. Among previously reported variants, a few proposed functional consequences have been reported. $14408 \mathrm{C}>\mathrm{T}$ within the RNA-dependent RNA polymerase gene (RdRp) may potentially affect proofreading or binding with other cofactors, thus affecting viral mutation rates (Pachetti et al., 2020). The variant $23403 \mathrm{G}>\mathrm{A}$ within the $\mathrm{S}$ protein has 


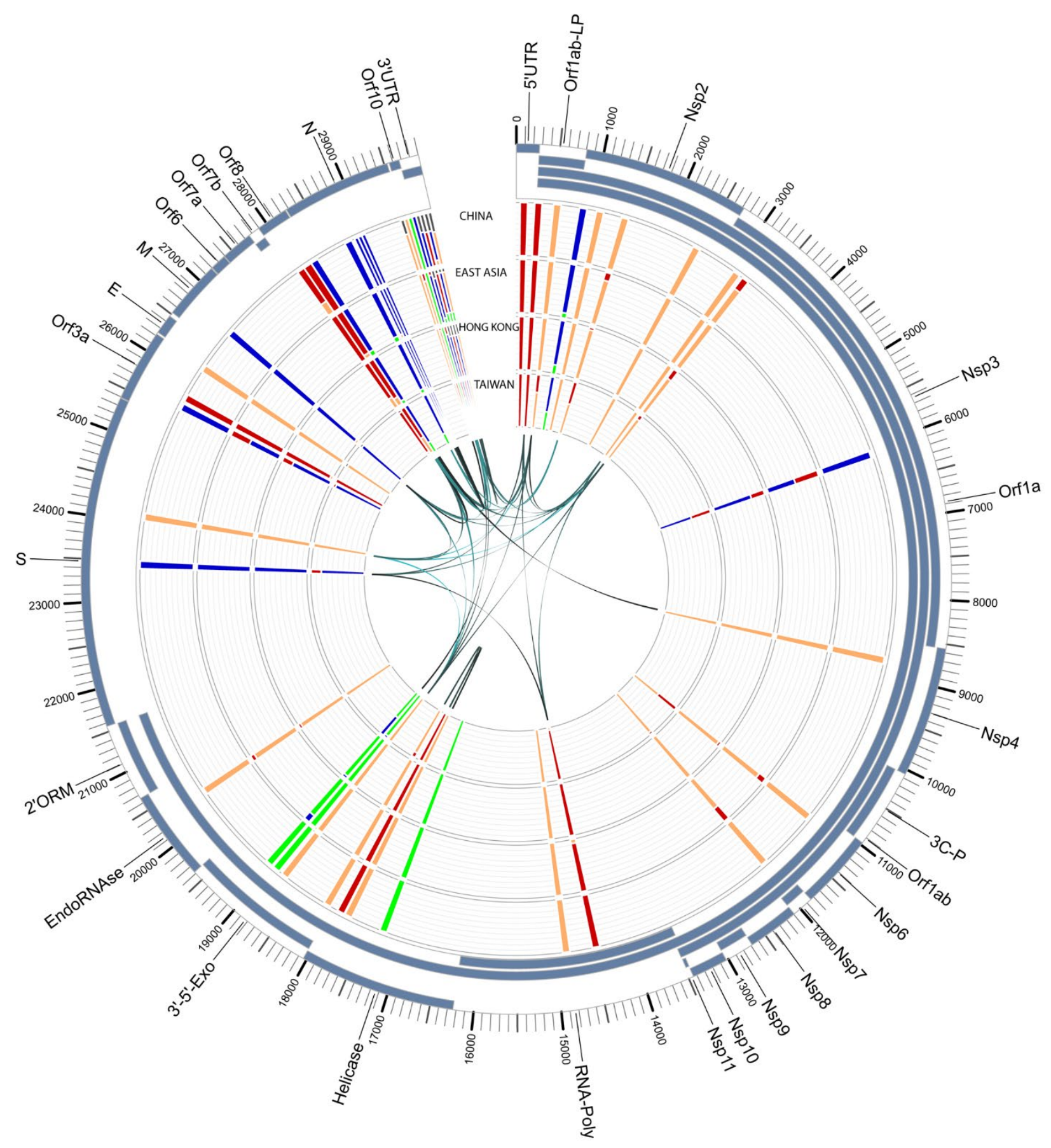

Figure 7. SARS-CoV-2 genome variants for China, East Asia, Hong Kong, and Taiwan. The outer track shows the gene/protein coding regions displayed in blue. The four inner tracks display nucleotide frequencies (green $(\mathrm{A})$, orange $(\mathrm{C})$, blue $(\mathrm{G})$, and red $(\mathrm{T})$ ) at 44 locations with an alternate allele frequency $>1 \%$. The inner-most track of arcs shows variant locations having a high linkage with $\mathrm{r}^{2}$ values ranging from 0.8 (narrow, light) to 1 (wide, dark). Abbreviations: leader protein (LP), $3 \mathrm{C}$-like-proteinase (3C-P), RNA-dependent-RNA-polymerase (RNA-Poly), 3'-to-5'-exonuclease (3'-5'-Exo), 2'-O-ribose-methyltransferase (2'ORM). 


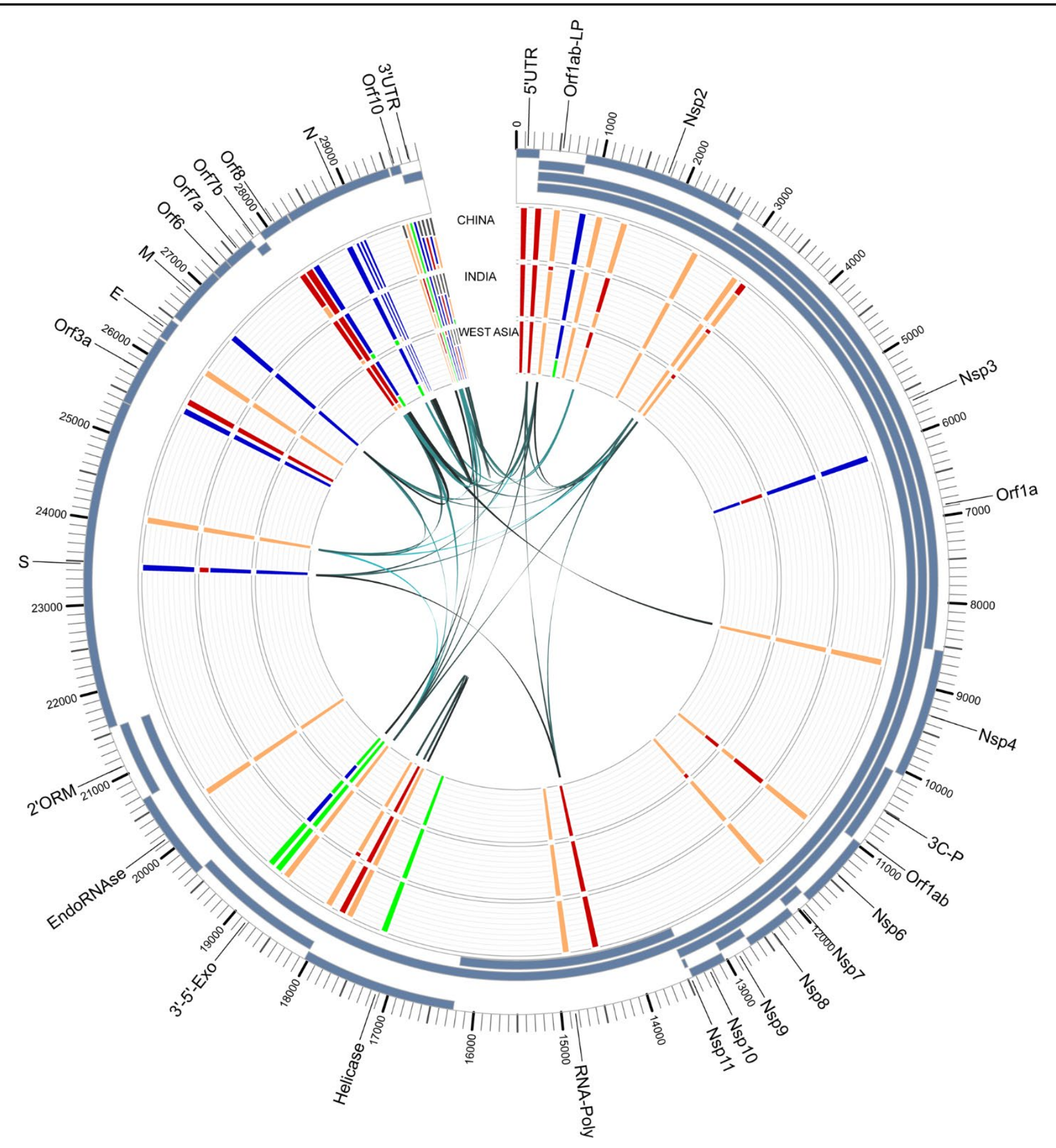

Figure 8. SARS-CoV-2 genome variants for China, India, and West Asia. The outer track shows the gene/protein coding regions displayed in blue. The four inner tracks display nucleotide frequencies (green (A), orange (C), blue $(\mathrm{G})$, and red (T)) at 44 locations with an alternate allele frequency $>1 \%$. The inner-most track of arcs shows variant locations having a high linkage with $\mathrm{r}^{2}$ values ranging from 0.8 (narrow, light) to 1 (wide, dark). Abbreviations: leader protein (LP), 3C-like-proteinase (3C-P), RNAdependent-RNA-polymerase (RNA-Poly), 3'-to-5'-exonuclease (3'-5'-Exo), 2'-O-ribose-methyltransferase (2'ORM). 


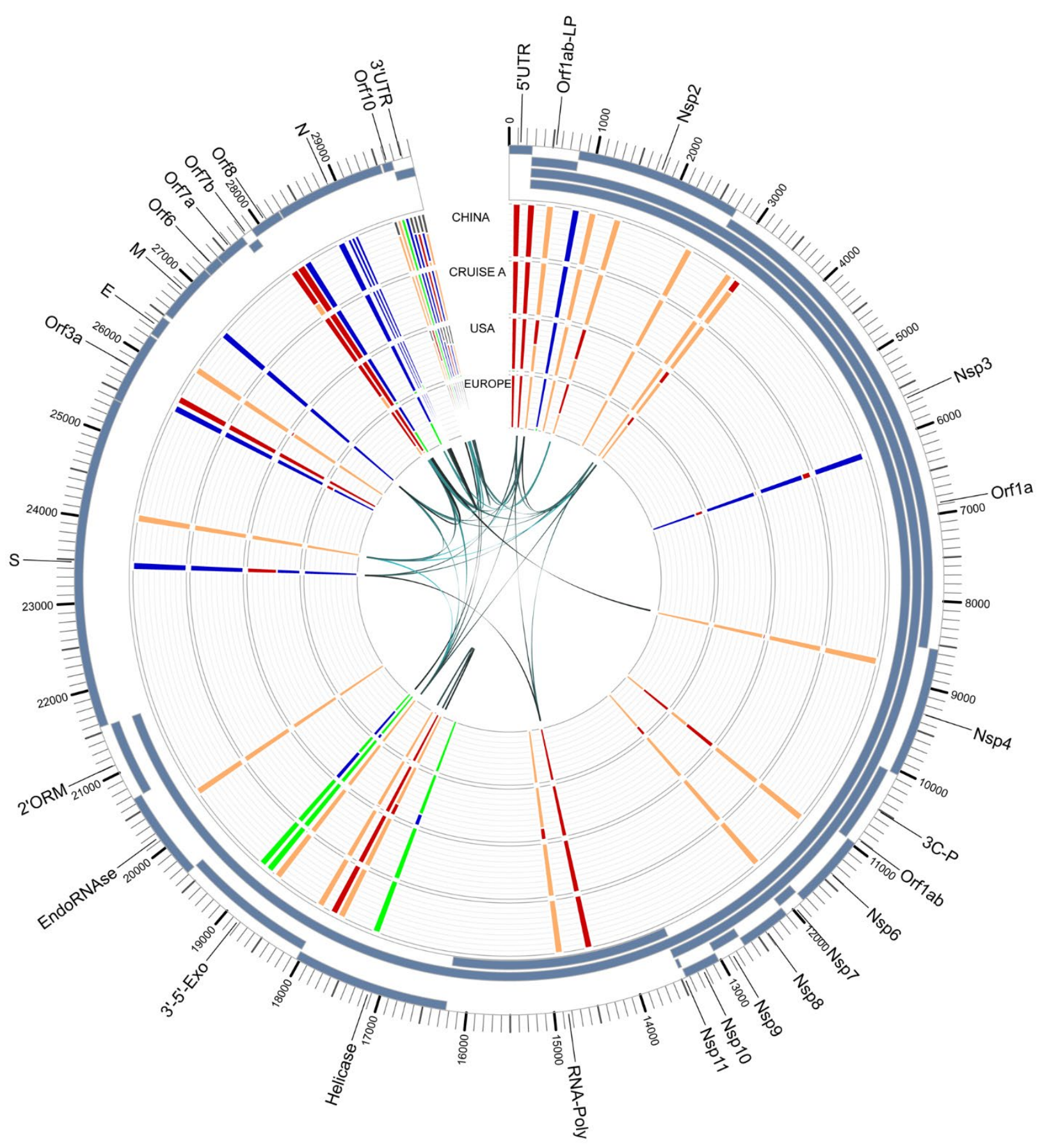

Figure 9. SARS-CoV-2 genome variants for China, CruiseA (Diamond Princess cruise docked in Oakland, CA), USA, and Europe. The outer track shows the gene/protein coding regions displayed in blue. The four inner tracks display nucleotide frequencies (green (A), orange (C), blue (G), and red (T)) at 44 locations with an alternate allele frequency $>1 \%$. The inner-most track of arcs shows variant locations having a high linkage with $\mathrm{r}^{2}$ values ranging from 0.8 (narrow, light) to 1 (wide, dark). Abbreviations: leader protein (LP), 3C-like-proteinase (3C-P), RNA-dependent-RNA-polymerase (RNA-Poly), 3'-to-5'-exonuclease (3'-5'-Exo), 2'-O-ribose-methyltransferase (2'ORM). 


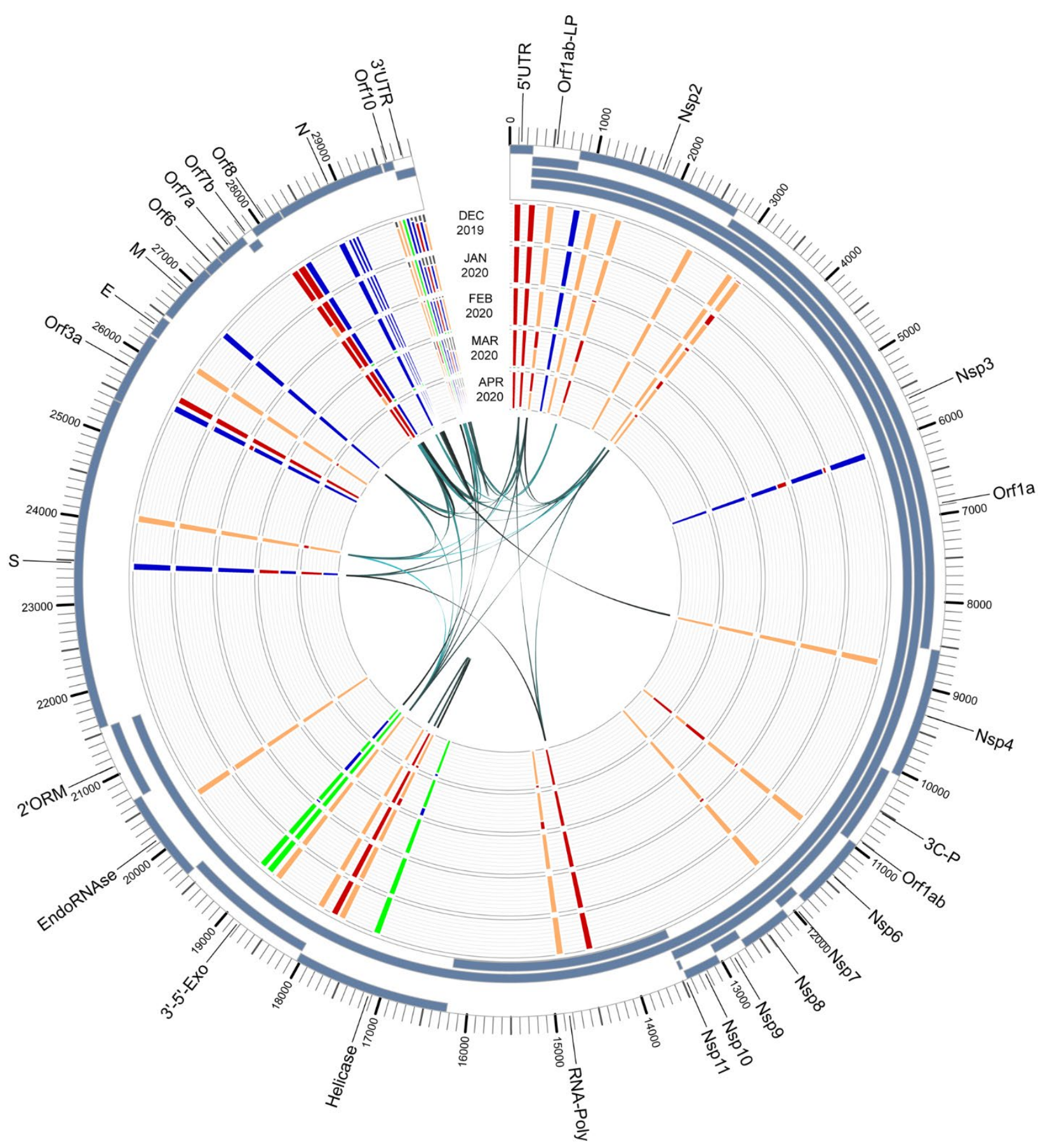

Figure 10. SARS-CoV-2 genome variants across time. Shown are the variants based on sampling date, ranging from December, 2019 to April, 2020 . The outer track shows the gene/protein coding regions displayed in blue. The four inner tracks display nucleotide frequencies (green (A), orange (C), blue (G), and red (T)) at 44 locations with an alternate allele frequency $>1 \%$. The inner-most track of arcs shows variant locations having a high linkage with $\mathrm{r}^{2}$ values ranging from 0.8 (narrow, light) to 1 (wide, dark). Abbreviations: leader protein (LP), 3C-like-proteinase (3C-P), RNA-dependent-RNA-polymerase (RNA-Poly), 3'-to-5'-exonuclease (3'-5'-Exo), 2'-O-ribose-methyltransferase (2'ORM). 
Table 5. Associations found with $r^{2}>0.8$ in Haploview.

\begin{tabular}{cc}
\hline Locations & $\mathbf{r}^{2}$ \\
\hline $490 / 3177$ & 1 \\
$8782 / 28144$ & 1 \\
$14408 / 23403$ & 1 \\
$17747 / 17858$ & 1 \\
$18998 / 29540$ & 1 \\
$26729 / 28077$ & 1 \\
$28882 / 28883$ & 1 \\
$241 / 14408$ & 0.997 \\
$241 / 23403$ & 0.997 \\
$28881 / 28882$ & 0.981 \\
$28881 / 28883$ & 0.981 \\
$29867 / 29868$ & 0.972 \\
$17858 / 18060$ & 0.968 \\
$17747 / 18060$ & 0.967 \\
$490 / 18736$ & 0.961 \\
$3177 / 18736$ & 0.961 \\
$18736 / 29700$ & 0.961 \\
$490 / 26729$ & 0.927 \\
$490 / 28077$ & 0.927 \\
\hline
\end{tabular}

\begin{tabular}{cc}
\hline Locations & $\mathbf{r}^{2}$ \\
\hline $3177 / 26729$ & 0.927 \\
$3177 / 28077$ & 0.927 \\
$490 / 29700$ & 0.923 \\
$3177 / 29700$ & 0.923 \\
$3037 / 14408$ & 0.916 \\
$3037 / 23403$ & 0.915 \\
$241 / 3037$ & 0.912 \\
$24034 / 26729$ & 0.901 \\
$24034 / 28077$ & 0.901 \\
$18736 / 26729$ & 0.891 \\
$18736 / 28077$ & 0.891 \\
$1397 / 28688$ & 0.874 \\
$1397 / 29742$ & 0.874 \\
$28688 / 29742$ & 0.861 \\
$26729 / 29700$ & 0.855 \\
$28077 / 29700$ & 0.855 \\
$490 / 24034$ & 0.835 \\
$3177 / 24034$ & 0.835 \\
$18736 / 24034$ & 0.802 \\
\hline
\end{tabular}

been hypothesized to increase transmissibility due to its expansion in global samples. This is thought to occur via one of two mechanisms, the first by diminishing interactions between the S1 and S2 promoters of the spike protein based on structural changes, and the second by affecting immunological response due to its location within an immune-dominant epitope (Korber et al., 2020).

Our analysis uncovered six previously unreported nonsynonymous variants, including $833 \mathrm{~T}>\mathrm{C}, 1059 \mathrm{C}>\mathrm{T}$, $11916 \mathrm{C}>\mathrm{T}, 18736 \mathrm{~T}>\mathrm{C}, 18998 \mathrm{C}>\mathrm{T}$, and $25563 \mathrm{G}>\mathrm{T}$. Five of these transition events occur within ORF1ab, and one occurs within ORF3a. Of these, two $(833 \mathrm{~T}>\mathrm{C}$ and $1059 \mathrm{C}>\mathrm{T}$ ) are within the nsp2 region, which is postulated to play a role in the host cell survival pathway via interactions with prohibitin (PHB) and prohbitin 2 (PHB2); one $(11916 \mathrm{C}>\mathrm{T})$ is within the $\mathrm{nsp} 7$ region that may act as a primase and therefore be involved in viral replication; two $(18376 \mathrm{~T}>\mathrm{C}$ and $18998 \mathrm{C}>\mathrm{T})$ are within the 3 ' to 5 ' exonuclease, which functions in proofreading; and one $(25563 \mathrm{G}>\mathrm{T})$ in ORF3a which forms viroporin ion channels and may modulate virus release (SwissProt, 2020).

While the SARS-CoV-2 virus is the product of a single copy RNA genome and our analysis of variants having a high association may be more applicable to eukaryotic genomes, it is known that recombination between strains is a key contributor to coronavirus evolution (Graham and Baric, 2010; Li et al., 2020b;Rehman et al., 2020). Korber, et al. (Korber et al., 2020) demonstrate that one particular mutation in the $\mathrm{S}$ protein $(\mathrm{S} 943 \mathrm{P})$ is likely a result of recombination between strains, due to the fact that at the time of reporting, it was found only in Belgium, but in multiple lineages, suggesting it was not a result of a founder sequence. Many associations have been identified (Korber et al., 2020;Pachetti et al., 2020; Tang et al., 2020;Yin, 2020), including the 38 we found with $r^{2}>0.8$. Further analysis of these is necessary, in order to rule out founder effect. A follow-up study using the reverse-genetic approach would be needed to understand the biological effects of the variant nucleotides and the nature of the high association between variant sequences (e.g. compensatory mutations) (Dinnon et al., 2020;Xie et al., 2020).

\section{Conclusion}

Our analysis resulted in 44 common variants within SARSCoV-2, 24 of which had not been previously described. From these variants, a total of 38 pairwise associations had an $\mathrm{r}^{2}$ value $>0.8$. Since COVID-19 is an emerging disease, a lot of current research efforts focused on understanding the origin and mutation of the SARS-CoV-2 virus and isolates. As a result, the amount of sequence information is increasing on a daily basis. In this study, we constructed a framework that will allow us to update our analysis rather efficiently with little intervention. We hope to expand our analysis to include more recent submissions to the NCBI GenBank database, as well as the GISAID EpiFlu database (Elbe and Buckland-Merrett, 2017). As more data becomes available, with additional isolates resulting from community-acquired transmission, a more complete picture of the evolution of SARS-CoV-2 will be possible.

\section{Conflict of Interest}

The authors declare that the research was conducted in the absence of any commercial or financial relationships that could be construed as a potential conflict of interest.

\section{$7 \quad$ Author Contributions}

ECR and DC contributed the overall study design. ECR and JHC implemented the algorithmic approaches. ECR wrote the first draft of the manuscript, and ECR and JHC produced the manuscript images. All authors contributed to manuscript revision, read and approved the submitted version.

\section{$8 \quad$ Funding}

This work was supported by the National Institutes of Health (NIH) grant P20GM103436. Its contents are solely the responsibility of the authors and do not represent the official views of the funding organization.

\section{$9 \quad$ Acknowledgements}

The authors wish to thank Jae Hwang and Juw Won Park for helpful discussions.

\section{References}

Andersen, K.G., Rambaut, A., Lipkin, W.I., Holmes, E.C., and Garry, R.F. (2020). The proximal origin of SARS-CoV-2. Nature medicine 26, 450-452. doi: 10.1038/s41591-020-0820-9

Atkins, J.F., Loughran, G., Bhatt, P.R., Firth, A.E., and Baranov, P.V. (2016). Ribosomal frameshifting and transcriptional slippage: from genetic steganography and cryptography to adventitious use. Nucleic acids research 44 , 7007-7078. doi: 10.1093/nar/gkw530 
Bar-On, Y.M., Flamholz, A.I., Phillips, R., and Milo, R. (2020). SARS-CoV-2 (COVID-19) by the numbers. arXiv preprint arXiv:2003.12886.

Barrett, J.C., Fry, B., Maller, J., and Daly, M.J. (2005). Haploview: analysis and visualization of LD and haplotype maps. Bioinformatics 21, 263-265. doi: 10.1093/bioinformatics/bth457

Chung, D., Rouchka, E.C., Ramirez, J., Palmer, K.E., Lasnik, A.B., Carrico, R., Arnold, F.W., Adcock, R.S., Chariker, J.H., Zhang, M., Alejandro, B., Wolf, L.A., and Waigel, S.Z., Wolfgang (2020). Characterizaton of a SARS-CoV-2 nasopharyngeal swab sample with an extremely high RNA load. Submitted.

Cohen, J., and Kupferschmidt, K. (2020). "Countries test tactics in 'war'against COVID-19". Science 367(6484):1287-1288. doi: 10.1126/science.367.6484.1287

Dinnon, K.H., Leist, S.R., Schafer, A., Edwards, C.E., Martinez, D.R., Montgomery, S.A., West, A., Yount, B.L., Hou, Y.J., and Adams, L.E. (2020). A mouseadapted SARS-CoV-2 model for the evaluation of COVID-19 medical countermeasures. bioRxiv. doi: 10.1101/2020.05.06.081497

Duffy, S. (2018). Why are RNA virus mutation rates so damn high? PLoS biology 16, e3000003. doi: 10.1371/journal.pbio.3000003

Elbe, S., and Buckland-Merrett, G. (2017). Data, disease and diplomacy: GISAID's innovative contribution to global health. Global Challenges 1, 33-46. doi: $10.1002 / \mathrm{gch} 2.1018$

Forster, P., Forster, L., Renfrew, C., and Forster, M. (2020). Phylogenetic network analysis of SARS-CoV-2 genomes. Proceedings of the National Academy of Sciences 117, 9241-9243. doi: 10.1073/pnas.2004999117

Graham, R.L., and Baric, R.S. (2010). Recombination, reservoirs, and the modular spike: mechanisms of coronavirus cross-species transmission. Journal of virology 84, 3134-3146. doi: 10.1128/JVI.01394-09

Johnson, M., Zaretskaya, I., Raytselis, Y., Merezhuk, Y., Mcginnis, S., and Madden, T.L. (2008). NCBI BLAST: a better web interface. Nucleic acids research 36 , W5-W9. doi: 10.1093/nar/gkn201

Khailany, R.A., Safdar, M., and Ozaslan, M. (2020). Genomic characterization of a novel SARS-CoV-2. Gene Reports, $100682 . \quad$ doi: 10.1016/j.genrep.2020.100682

Korber, B., Fischer, W., Gnanakaran, S.G., Yoon, H., Theiler, J., Abfalterer, W., Foley, B., Giorgi, E.E., Bhattacharya, T., and Parker, M.D. (2020). Spike mutation pipeline reveals the emergence of a more transmissible form of SARSCoV-2. bioRxiv. doi: 10.1101/2020.04.29.069054

Lassmann, T. (2020). "Kalign 3: multiple sequence alignment of large datasets". Bioinformatics 36(6), 1928-1929. doi: 10.1093/bioinformatics/btz795

Li, R., Pei, S., Chen, B., Song, Y., Zhang, T., Yang, W., and Shaman, J. (2020a). Substantial undocumented infection facilitates the rapid dissemination of novel coronavirus (SARS-CoV-2). Science 368, 489-493. doi: 10.1126/science.abb3221

Li, X., Giorgi, E.E., Marichann, M.H., Foley, B., Xiao, C., Kong, X.-P., Chen, Y., Korber, B., and Gao, F. (2020b). Emergence of SARS-CoV-2 through Recombination and Strong Purifying Selection. bioRxiv. doi: 10.1101/2020.03.20.000885
Pachetti, M., Marini, B., Benedetti, F., Giudici, F., Mauro, E., Storici, P., Masciovecchio, C., Angeletti, S., Ciccozzi, M., and Gallo, R.C. (2020). Emerging SARS-CoV-2 mutation hot spots include a novel RNA-dependentRNA polymerase variant. Journal of Translational Medicine 18, 1-9. doi: 10.1186/s12967-020-02344-6

Phan, T. (2020). Genetic diversity and evolution of SARS-CoV-2. Infection, Genetics and Evolution 81, 104260. doi: 10.1016/j.meegid.2020.104260

Rehman, S.U., Shafique, L., Ihsan, A., and Liu, Q. (2020). Evolutionary trajectory for the emergence of novel coronavirus SARS-CoV-2. Pathogens 9, 240. doi: 10.3390 /pathogens 9030240

Sayers, E.W., Cavanaugh, M., Clark, K., Ostell, J., Pruitt, K.D., and KarschMizrachi, I. (2019). GenBank. Nucleic acids research 47, D94-D99. doi: 10.1093/narlgky989

Shen, Z., Xiao, Y., Kang, L., Ma, W., Shi, L., Zhang, L., Zhou, Z., Yang, J., Zhong, J., and Yang, D. (2020). Genomic diversity of SARS-CoV-2 in Coronavirus Disease 2019 patients. Clinical Infectious Diseases, ciaa203. doi: $10.1093 / \mathrm{cid} / \mathrm{ciaa} 203$

Swissprot (2020). Severe acute respiratory syndrome coronavirus 2 (SARS-CoV-2) [Online]. Available: https://swissmodel.expasy.org/repository/species/2697049 [Accessed 5/19/2020 2020].

Tang, X., Wu, C., Li, X., Song, Y., Yao, X., Wu, X., Duan, Y., Zhang, H., Wang, Y., and Qian, Z. (2020). On the origin and continuing evolution of SARS-CoV-2. National Science Review, nwaa036. doi: 10.1093/nsr/nwaa036

Wang, C., Liu, Z., Chen, Z., Huang, X., Xu, M., He, T., and Zhang, Z. (2020a). The establishment of reference sequence for SARS-CoV-2 and variation analysis. Journal of medical virology 92, 667-674. doi: 10.1002/jmv.25762

Wang, M., Li, M., Ren, R., Brave, A., Van Der Werf, S., Chen, E.-Q., Zong, Z., Li, W., and Ying, B. (2020b). International expansion of a novel SARS-CoV-2 mutant. medRxiv. doi: 10.1101/2020.03.15.20035204

World Health Organization. (2020). Coronavirus Disease (COVID-19) Pandemic [Online]. Available: https://www.who.int/emergencies/diseases/novelcoronavirus-2019 [Accessed 05/11/2020 2020].

Xie, X., Muruato, A., Lokugamage, K.G., Narayanan, K., Zhang, X., Zou, J., Liu, J., Schindewolf, C., Bopp, N.E., and Aguilar, P.V. (2020). An Infectious cDNA Clone of SARS-CoV-2. Cell Host \& Microbe 27, 841-848. doi: 10.1016/j.chom.2020.04.004

Yin, C. (2020). Genotyping coronavirus SARS-CoV-2: methods and implications. Genomics. doi: 1016/j.ygeno.2020.04.016.

Zhu, N., Zhang, D., Wang, W., Li, X., Yang, B., Song, J., Zhao, X., Huang, B., Shi, W., and $\mathrm{Lu}, \mathrm{R}$. (2020). A novel coronavirus from patients with pneumonia in China, 2019. New England Journal of Medicine, 382:727-733. doi: 10.1056/NEJMoa2001017 